\title{
Temporal characteristics of primary-secondary message interference in a dichotic listening task
}

\author{
BRUCE A. AMBLER and SEBASTIANO A. FISICARO \\ University of Texas, Arlington, Texas 76019 \\ and \\ ROBERT W. PROCTOR \\ Michigan State University, East Lansing, Michigan 48829
}

\begin{abstract}
Previous experiments have shown that during performance of a shadowing task the unattended message is processed at a semantic level. Four experiments are reported that deal with the question of how processing of the unattended message influences the difficulty of shadowing the attended message. Experiment I demonstrated that pupil dilation measures the difficulty associated with a shadowing task. In Experiment II it was shown that during shadowing of 20 -item lists pupil dilation decreases as shadowing continues when either words or white noise are in the unattended channel. These results support the idea that it takes time for the attentional mechanism to focus on the attended message. In Experiments III and IV it was found that this decrease in difficulty of shadowing is much less when the attended and unattended messages come from the same stimulus category (letter-letter, digit-digit). The results indicate that, when the unattended message is from the same stimulus category as the attended message, it can interfere with the ability of the attentional system to focus on the attended message.
\end{abstract}

Shadowing, a task which consists of listening to a continuous message and concurrently repeating it aloud, involves both input and output operations. When subjects are given two messages at the same time but are asked to shadow only one, successful performance of the shadowing task is used as a demonstration of selective attention. The early results from such experiments indicated that the messages could be separated on the basis of physical characteristics, such as vocal frequency and auditory spatial location (Treisman, 1969). Many studies have reported that subjects remembered very little about the message that had not been shadowed. However, other evidence has indicated that this unattended message was in fact being processed. For example, Moray (1959) found that subjects responded to their name when it was presented in the unattended message. More recent evidence has also indicated that unattended-message items can be processed at a semantic level, even when there is no requirement to do so. Corteen and Dunn (1974) and Corteen and Wood (1972) classically conditioned a GSR response to a specific set of words. In a subsequent shadowing task, the conditioned stimuli were presented in the secondary message. A conditioned response occurred even though subjects reported not having been aware of the presentation of the conditioned stimuli.

Given that information in the unattended message

Requests for reprints should be sent to Bruce A. Ambler, Department of Psychology, University of Texas at Arlington, Arlington, Texas 76019. This research was supported by funds provided by the Organized Research Committee of the University of Texas at Arlington. is processed, the question then arises as to how processing of the unattended (irrelevant) message influences processing of the attended (relevant) meassage. Lewis (1970) studied the influence of the unattended message on the processing of the attended message by measuring vocal reaction time to shadowed material (words) while varying characteristics of the unattended message. He found that vocal reaction time was longer when the paired word in the unattended message was a synonym than when it is not.

Treisman, Squire, and Green (1974) proposed that the degree to which the unattended message interferes with the attended message might vary with time. They argued that it could take time for attention to become focused on the relevant message. Support for this was obtained by replicating Lewis' (1970) results and finding that the semantic interference he observed occurred primarily at the beginning of each list.

The Lewis (1970) and Treisman et al. (1974) results were obtained using vocal reaction time as a measure of the difficulty of performing the shadowing task. The present study continues this line of research using a different measure of task difficulty, pupil dilation. Pupil dilation has been shown to reflect momentary mental effort (see Goldwater, 1972, and Kahneman, 1973, for detailed reviews of the literature). For example, Hess and Polt (1964) found that subjects' pupil size increased as the difficulty of the multiplication problems they were solving increased. Furthermore, Beatty and Kahneman (1966) and Kahneman and Beatty (1966) found that pupil dilation increased with increases in memory load. However, it has not yet been 
shown that pupil dilation reflects the amount of cognitive load imposed by a shadowing task. If pupil dilation does reflect the difficulty of a shadowing task, then it could provide a useful methodology for studying the influence of an unattended message on the difficulty of processing the attended message. Experiment I was performed as an initial investigation of the relationship between shadowing and pupil dilation.

\section{EXPERIMENT I}

Zelniker (1971) compared the attentional requirements of two tasks. One involved shadowing, the other did not. She employed delayed auditory feedback (DAF) in an attempt to show that irrelevant input is processed less as more attention is directed elsewhere (as in the shadowing task). Zelniker used stuttering frequency resulting from DAF as a measure of the degree to which the DAF was analyzed.

Subjects were required to listen to three pairs of twodigit numbers presented to one ear at the rate of two two-digit numbers per second. In the nonshadow condition, subjects were asked to listen to the first pair of two-digit numbers and then to repeat it aloud twice, coinciding with the presentation of the second and third pairs of two-digit numbers, respectively. In the shadow condition, subjects were asked to listen to the first pair of two-digit numbers, repeat them aloud while listening to the second pair of two-digit numbers, and then to repeat aloud the second pair of two-digit numbers while hearing the third pair of two-digit numbers. For example, assume the subject was presented with $52-89 / 65-24 / 38-41$. In the nonshadow condition, the subject would listen to $52-89$, then repeat $52-89$ aloud twice, once while hearing 65-24 and once while hearing 3841 . In the shadow condition, the subject would repeat $52-89$ aloud while listening to $65-24$, and repeat 65-24 aloud while hearing 38-41. Subjects' responses were recorded, delayed $.2 \mathrm{sec}$ and then played back to the subject in the other ear during the task. Thus, the DAF served as the unattended message.

Stuttering frequency under DAF was greater in the nonshadow condition than in the shadow condition. Thus, Zelniker (1971) showed that the shadow task required more attention that the nonshadow task.

In Experiment $I$, the shadow and nonshadow conditions were compared to see if the additional attention required by the shadowing task would result in greater pupil dilation. If so, pupil dilation would be established as being sensitive to differences in attentional task demands.

\section{Method}

Subjects. The subjects were four graduate students and four undergraduate volunteers from an introductory course in psychology.

Stimulus materials. Two lists of 15 stimulus sets were constructed in the following manner: Two-digit numbers were selected from a table of random numbers and grouped into three pairs of two-digit numbers. The following restriction was imposed: Each two-digit number had to contain exactly three syllables. Thus, the following numbers were not used: (a) numbers below 21 and (b) numbers which contained a zero or a seven. Furthermore, no digit could appear more than once within any pair of two-digit numbers (i.e., 22-89/ and 28-92/ were not allowed). A digit could appear twice in a stimulus set (three pairs of two-digit numbers), but none of the two-digit numbers could begin or end with the same digit (i.e., 28-49/25-63/96-34 was not allowed because 28 and 25 each begin with a 2 , and 84-63/91-45/23-58 was not allowed because 63 and 23 each end with a 3 ).

Stimuli were taped on a recorder in a male voice at the rate of two two-digit numbers per second. Thus, each stimulus trial lasted $3 \mathrm{sec}$. The intertrial in terval (TTI) was $15 \mathrm{sec}$ and each subject received 30 trials (15 nonshadow trials and 15 shadow trials).

Apparatus. Stimuli were presented via stereophonic tape recorder, through both external speakers and stereophonic headphones which were worn by the subject. The subject was positioned in a standard classroom desk on which a chin and forehead rest had been mounted. By pressing on the forehead rest, the subject was able to respond verbally without changing the position of his eye. Pupil dilations were continuously monitored and recorded for each subject via video camera. The video camera lens was completely covered by a sheet of white typewriter paper $\left(8 \frac{1}{2} \times 11 \mathrm{in}\right.$.) except for a small circular hole ( $1 / 2$ in. in diam) in the center. With the use of extension rings, it was situated directly in front of the subject's right eye, 1 in. away. This arrangement effectively eliminated extraneous distractions and the reflection of the camera lens from the subject's eye, and resulted in a $17 \mathrm{X}$ enlargement of the pupil on the video monitor. The external speakers were placed in front of a microphone connected directly to the video tape recorder so that stimuli and pupil dilations could be recorded simultaneously.

Design and procedure. A within-subjects 2 by 2 factorial design was employed. The first factor consisted of the two conditions, shadow vs. nonshadow. The other factor included the two positions within the task where pupil size was measured-at the second half of the second pair of numbers (Position 1) and at the second half of the third pair of numbers (Position 2). Thus, if 52-89/63-45/21-38 were presented, measurements would be taken when the subject was hearing 45 and 38 . Two sites were selected in order to increase the probability of measuring pupillary dilations resulting from actual task demands.

Half of the subjects were given nonshadow instructions, presented with 15 nonshadow trials, given shadow instructions, and then presented with 15 shadow trials. The other half of the subjects went through the shadow condition before the nonshadow condition. The experimenter noted on a response sheet which trials the subject successfully completed.

Pupil dilation measurement. The video tape (containing both the actual stimuli presented to the subjects and a record of pupil dilations) was played back after the experimental session. At least two experimenters were present whenever measurements were taken. One controlled the playback of the video tape and the other both consulted the response sheet, which indicated correct and incorrect trials, and recorded the data. A calibrated transparent grid was placed over the video monitor and measurements of pupil size were made. Data were collected from the last 10 correct trials in each of the two conditions. Thus, the first few trials were treated as practice (although subjects were not told this). No measurements were taken for incorrect trials. Within each correct trial, four pupil dilation measures were taken in the following manner: Two baseline measures were taken during the 15 -sec ITI. The first was taken $7 \mathrm{sec}$ after the presentation of the last two-digit number of the previous stimulus; the second was taken $5 \mathrm{sec}$ after the first $(12 \mathrm{sec}$ after the last two-digit number). The tape was stopped at Position 1 and then 
at Position 2, where measurements were taken. For each subject, means for the 20 baseline measures, the 10 Postition 1 measures, and the 10 Position 2 measures were computed for each condition. Then the mean baseline was subtracted from each of the position means. The analysis of variance was performed on the mean difference scores.

\section{Results and Discussion}

The mean difference scores for pupil size are presented in Table 1 . The 2 by 2 analysis of variance produced the following results: The effect of Attention was significant $[F(1,7)=17.67, p<.001, \mathrm{MSe}=.016]$. This indicates that the shadow task resulted in greater pupil dilation than the nonshadow task. The effect of Position was not significant $[F(1,7)=1.34, p>.05$, $\mathrm{MSe}=.032]$. This indicates no difference in the measurements taken at the two positions. Finally, the Attention by Position interaction was not significant $[F(1,7)<1, \mathrm{MSe}=.007]$. This suggests no differential effects of Attention condition on Position of measurement.

The main result of the experiment was that pupil dilation was greater when subjects had to both input and output information at the same time (shadow condition) than when they only had to output (nonshadow condition). The condition which Zelniker (1971) found to reduce stuttering by requiring more attention (shadow condition) was found to increase pupil dilation. This supports the notion that the amount of attention involved in a shadowing task is reflected in a measure of pupil dilation.

It should be noted that Kahneman (1973) explained the Zelniker (1971) results by using a capacity-allocation model of selective attention. He argued that more capacity is required by the shadow task, thus leaving less spare capacity which can be used to analyze secondary messages. Furthermore, he proposed that pupil dilation is a measure of the amount of capacity utilized by a task. The present finding that pupil dilation is greater for the shadow task than for the nonshadow task is consistent with this interpretation.

\section{EXPERIMENT II}

In Experiment I the digit lists were quite short and there were no secondary (irrelevant) messages. In this experiment subjects were required to shadow longer lists of digits. This was done to see if interference from secondary messages occurs primarily at the beginning of lists, as Treisman et al. (1974) suggest. The nature of the shadowed material (one- vs. two-digit num-

Table 1

Experiment I: Mean Difference in Pupil Size (Millimeters) as a Function of Position of Measurement and Attention Condition

\begin{tabular}{lcc}
\hline & Position 1 & Position 2 \\
\hline Nonshadow Condition & .42 & .49 \\
Shadow Condition & .61 & .68 \\
\hline
\end{tabular}

bers) was varied to check for its effect on shadow task difficulty. Another factor which was varied was the type of material presented in the unattended ear, a list of unrelated words vs. white noise of equal loudness. If having words in the unattended channel increases task difficulty, then this should result in an increase in pupil size.

\section{Method}

Subjects. The subjects were nine undergraduate volunteers from an introductory course in psychology.

Stimulus materials. Twenty lists of digits were compiled using a table of random numbers. Half of the lists contained 20 two-digit numbers and half contained 20 one-digit numbers. The stimuli were taped in a male voice at the rate of one number (either one- or two-digit) per second at $68 \mathrm{~dB}$. For half of the lists, white noise of $68 \mathrm{~dB}$ was played on the other channel. For the other lists, 20 one-syllable words were recorded at a level of $68 \mathrm{~dB}$ in such a manner that they coincided with the presentation of each number in the primary message. There was a 15-sec ITI between lists.

Apparatus. The apparatus was the same as that used in Experiment 1 .

Design and procedure. A within-subjects 2 by 2 by 5 factorial design was employed. The first factor consisted of the number of digits (one vs. two) in the numbers that were being shadowed. The second factor was whether the unattended channel contained words or noise. The third factor consisted of five different positions throughout each list where pupil dilation measurements were made.

Eight of the 20 lists (four two-digit and four one-digit) were used for shadow practice. No unattended message was given during presentation of these lists. The last 12 lists were considered experimental trials. Half of the six one-digit lists and half of the six two-digit lists were paired with words, while the rest were paired with noise. This resulted in three different lists being presented in each of the four attended-unattended message combinations. The 12 lists were presented in a random order.

Subjects were instructed to shadow (i.e., repeat aloud) the numbers as they heard them, while ignoring anything that might be presented to the other ear. Five of the subjects received the relevant messages on the left ear and the irrelevant messages on the right ear. The reverse was the case for the other four subjects.

Pupil dilation measurement. Pupil dilation measures were made using the same equipment and procedures as in Experiment I. Trial measures were taken at the 4 th, 8 th, 12 th, 16th and 20 th positions in each list. Two baseline measures were taken just prior to the presentation of each list, one $10 \mathrm{sec}$ before and one $5 \mathrm{sec}$ before. Since each subject received 12 lists (three lists in each of four conditions), there were six baseline measures per condition. In each condition the six baselines were averaged, and the three trial measures were averaged at each of the five positions. The mean baseline was subtracted from each of the five position means in each condition; these mean difference scores were used in the analysis.

\section{Results and Discussion}

Pupil dilation differences for eight subjects were analyzed. ${ }^{1}$ The mean pupil dilation differences are presented in Figure 1. The analysis showed a clear effect of Position $[\mathrm{F}(4,28)=14.77, \mathrm{p}<.001, \mathrm{MSe}=.028]$, with pupil size decreasing over time. The significant Position effect is consistent with the proposal of Treisman et al. (1974) that interference decreases over time because it takes time for attention to become 


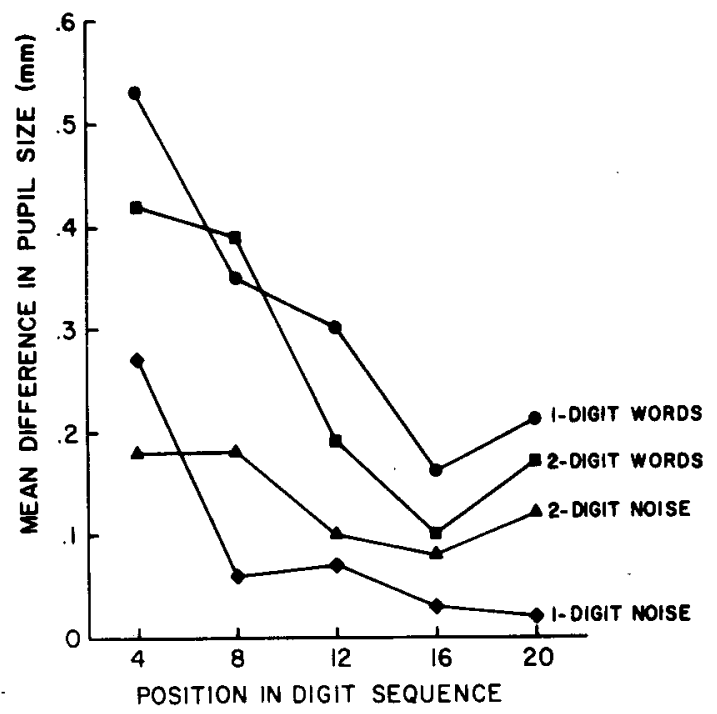

Figure 1. Mean difference from baseline in pupil size (millimeters) as a function of position of measurement in digit sequence for primary (one vs. two digits)/secondary (noise vs. words) message combinations.

focused on the relevant message. The difference between the two unattended messages was also significant $[F(1,7)=15.03, p<.01, \mathrm{MSe}=.074]$. Words resulted in greater difficulty than noise: There was no effect for number of digits shadowed $[F(1,7)<1.0$, $\mathrm{MSe}=.088]$.

The Position by Type of Unattended Message interaction was significant $[F(4,28)=3.06, p<.05$, $\mathrm{MSe}=.013 \mathrm{~J}$. This indicates that the decrease across positions was greater when words were the unattended message than when noise was the unattended message; this appears to be due to the fact that the word curves started higher. The Position by Type of Attended Message interaction was not significant $[\mathrm{F}(4,28)=1.25$, $\mathrm{p}>.05, \mathrm{MSe}=.016 \mathrm{]}$, and neither the Unattended by Attended Message interaction $[\mathrm{F}(1,7)=1.05, \mathrm{p}>.05$, $\mathrm{MSe}=.157]$ nor the three-way interaction $[\mathrm{F}(4,28)<1.0, \mathrm{MSe}=.010]$ was significant.

Even though the error rates for this experiment were very low, they were also analyzed (see Table 2). The 20 trials for each list were divided into four quadrants and errors were computed separately for each quadrant in each of the four conditions. The quadrant differences were taken to be analogous to the position differences in the pupil dilation measure. A within-subjects 2 by 2 by 4 analysis of variance (number of digits by type of unattended message by quadrant) was performed on the error rate data. None of the resulting Fs approached significance.

\section{EXPERIMENT III}

Experiment II demonstrated that relatively gross differences in the content of the secondary message produce significant differences in the difficulty of a shadowing task. That is, when the unattended message was comprised of patterned stimuli (words), the shadow. ing task was more difficult than when it was not (noise). Experiment III was designed to determine whether shadowing-task difficulty is sensitive to finer differences in the relationship of the secondary message to the primary message. This was done by varying the category from which items were drawn. Specifically, the primary messages were composed of letters and the secondary messages were composed of letters, digits, words, or prose. If category similarity influences shadowing difficulty, then greater pupil dilation should be observed when the unattended message is composed of letters than when it is not.

However, as mentioned previously, there is evidence to suggest that the unattended message is processed at a semantic level (e.g., Corteen \& Wood, 1972) and that another factor which may affect shadowing difficulty is the level of semantic content of the unattended message. In other words, secondary messages which have higher degrees of semantic complexity may interfere more with shadowing than secondary messages which have relatively lower degrees of semantic complexity. In this experiment prose was assumed to involve more semantic complexity than words, and words more than either digits or letters.

\section{Method}

Subjects. Ten volunteers from undergraduate psychology courses served as subjects.

Stimulus materials. Twenty-eight different lists of 20 letters each (excluding $W$ ) were randomly constructed such that no letter appeared more than once in a list and each list was unique. Four lists were constructed from each of the following stimuli: prose, digits, and words. The prose lists consisted of four different passages from novels. Four digit lists of 20 numbers each were compiled using a table of random numbers. Both oneand two-digit numbers were used, but no number could appear more than once in a list. The word lists consisted of 80 onesyllable words randomly divided into four lists of 20 words each. All lists were recorded in the same male voice at the rate of one item per second, except for the prose, which was read for $20 \mathrm{sec}$. The intertrial interval (ITI) between lists was $15 \mathrm{sec}$.

Nine of the letter lists were used for shadow practice. One of each of the letter, word, digit, and prose lists was used as a listening control. Dichotic lists were constructed from the remaining stimuli. Three of the remaining 18 letter lists and the remaining three prose, word, and digit lists were used as secondary messages. The last 15 letter lists were used as primary (shadow) messages. The primary and secondary messages were randomly paired and taped together on separate tracks in such a manner that irrelevant items coincided with the presentation of the relevant items. The prose was read for $20 \mathrm{sec}$, so that it matched primary message duration. Three letter lists were paired with white noise. ${ }^{2}$

Table 2

Experiment 11: Percent Errors

\begin{tabular}{|c|c|c|c|c|c|c|c|c|}
\hline \multirow{3}{*}{$\begin{array}{c}\text { Unattended } \\
\text { Message }\end{array}$} & \multicolumn{4}{|c|}{1 Digit } & \multicolumn{4}{|c|}{2 Digit } \\
\hline & \multirow{2}{*}{\multicolumn{3}{|c|}{$\begin{array}{rr}\text { Quadrant } \\
2 & 3\end{array}$}} & \multirow[b]{2}{*}{4} & \multicolumn{4}{|c|}{ Quadrant } \\
\hline & & & & & 1 & 2 & 3 & 4 \\
\hline Words & .57 & .00 & .57 & .57 & 1.10 & .28 & .57 & .28 \\
\hline Noise & .00 & .00 & .28 & .28 & .28 & .00 & .57 & .83 \\
\hline
\end{tabular}


The range of intensity measured within each primary message was $68-72 \mathrm{~dB}$. Within each secondary message the intensity range was $70-74 \mathrm{~dB}$.

Apparatus. The apparatus was basically the same as that used in Experiment $I$.

Design and procedure. A within-subjects 4 by 5 factorial design was used. The first factor consisted of the four types of irrelevant input: prose, words, digits, and letters. The second factor consisted of the five positions of measurement.

Subjects were first given nine shadow-practice trials. They were then given the four control lists and told that these were like the messages they would be hearing in the irrelevant ear. They were instructed to just listen and not repeat anything. Pupillary dilations were not recorded during shadow practice. Video taping began with the presentation of the control lists. Next, subjects were given 15 dichotic shadowing trials. Each primary message was preceded by "one, two, three" to facilitate the beginning of shadowing. Half of the subjects received primary messages in the left ear, while the rest received them in the right ear. All subjects were instructed to shadow the letters and ignore the secondary message. The prose, word, letter, digit, and noise lists were randomly presented to subjects, with the constraint that no two lists of the same type of irrelevant input could follow each other.

Pupil dilation measurement. The pupil dilation measures were made in the same manner as those in Experiment II. The sole difference was that subjects received 15 lists (three lists in each of five conditions) instead of 12 .

\section{Results}

The mean differences from baseline for the prose, word, letter, and digit conditions are shown in Figure 2. The effect of Position was significant $[F(4,36)=8.00$, $p<.001, \mathrm{MSe}=.045]$. There was no effect of Seconday Message Type $[\mathrm{F}(3,27)=1.11, \mathrm{p}>.05, \mathrm{MSe}=$ .113]. However, there was a significant Position by Secondary Message Type interaction $[F(12,108)=2.33$, $\mathrm{p}<.025, \mathrm{MSe}=.013]$.

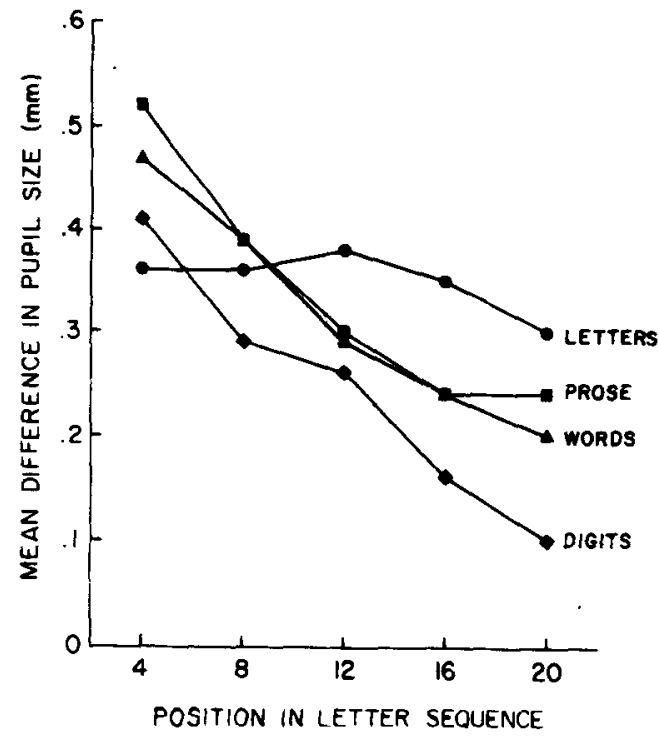

Figure 2. Mean difference from baseline in pupil size as a function of position of measurement in letter sequence, with letters as the primary message and letters, prose, words, or digits as the secondary message.
Table 3

Experiment III: Percent Errors

\begin{tabular}{lcccc}
\hline & \multicolumn{4}{c}{ Quadrant } \\
\cline { 2 - 5 } $\begin{array}{l}\text { Unattended } \\
\text { Message }\end{array}$ & 1 & 2 & 3 & 4 \\
\hline Prose & 4.0 & 0.0 & 4.6 & 4.0 \\
Words & 4.6 & 4.6 & 7.3 & 5.3 \\
Digits & 4.0 & 5.3 & 1.3 & 1.3 \\
Letters & 6.6 & 4.6 & 8.0 & 7.3 \\
\hline
\end{tabular}

The main effect for Position indicates that pupil size decreased over time. The source of the interaction was revealed by partitioning the sum of squares into two components. The first component, which compared the letter curve to the other three curves, was significant $[F(4,108),=6.25, p<.001, \mathrm{MSe}=.013]$. This indicates that the letter curve decreased in a nianuer which differed from the other curves. The second component, which was merely the residual sum of squares when the sum of squares for the first component was accounted for, was not significant $[F(8,108)<1.0$, $\mathrm{MSe}=.013]$. This means that the prose, digit, and word curves did not differ in the manner in which they decreased.

A separate analysis was performed on error scores. Each list of 20 letters was divided into four quadrants of five letters each, and errors were scored separately within each quadrant. The percentage of errors for each quadrant in each condition is presented in Table 3. A within-subjects 4 by 4 (quadrant by secondary message type) analysis of variance resulted in none of the Fs approaching significance. This means that the observed differences in pupil dilation could not be the result of an attention-accuracy tradeoff.

The control condition, during which subjects just listened to the four secondary messages, was also analyzed for the four different secondary verbal messages. For each condition one mean was computed from the five measures taken during list presentation and another mean was computed from the two baseline measures taken before list presentation. The difference between these means was used in a within-subjects one-way analysis of variance. No difference in pupil dilation was found for the four types of secondary verbal message $[F(3,27)=1.16, p>.05, \mathrm{MSe}=.051]$. This indicates that the secondary messages did not differ in arousal content.

\section{Discussion}

The results of Experiment II were replicated. Shadowing difficulty as measured by pupil dilation decreased over time. However, an effect for different levels of content of the unattended messages was not found. There was no overall difference in shadowing difficulty when prose, words, letters, or digits comprised the unattended message.

The most interesting result of Experiment III con- 
cerned the decrease in pupil dilation across positions as shadowing proceeded. A significant effect for position was obtained with verbal unattended messages, as in Experiment II. However, among the verbal conditions there was an interaction due to the fact that the rate of decrease in difficulty of shadowing was significantly less when letters comprised the secondary message. As shadowing continues, difficulty decreases if the two messages come from different semantic categories. However, if they come from the same category, decreases in shadowing difficulty are at best small and perhaps even nonexistent. Thus, category similarity results in more interference, while semantic complexity of the secondary message does not.

\section{EXPERIMENT IV}

Experiment IV was a replication of Experiment III using digits as the attended message rather than letters. If category similarity is actually the factor influencing the rate of decrease of shadowing difficulty, then a minimal rate of decrease should be found when digits comprise the unattended message.

\section{Method}

Subjects. The subjects were nine undergraduate volunteers from an introductory psychology course.

Procedure. The apparatus and procedure were the same as those used in Experiment III. The only difference was that the attended message consisted of two-digit numbers. Two-digit numbers were also used in the unattended message.

\section{Results}

The data were analyzed in the same way as in Experiment III. ${ }^{2}$ As seen in Figure 3, pupil dilation data produced results very similar to those of Experiment III.

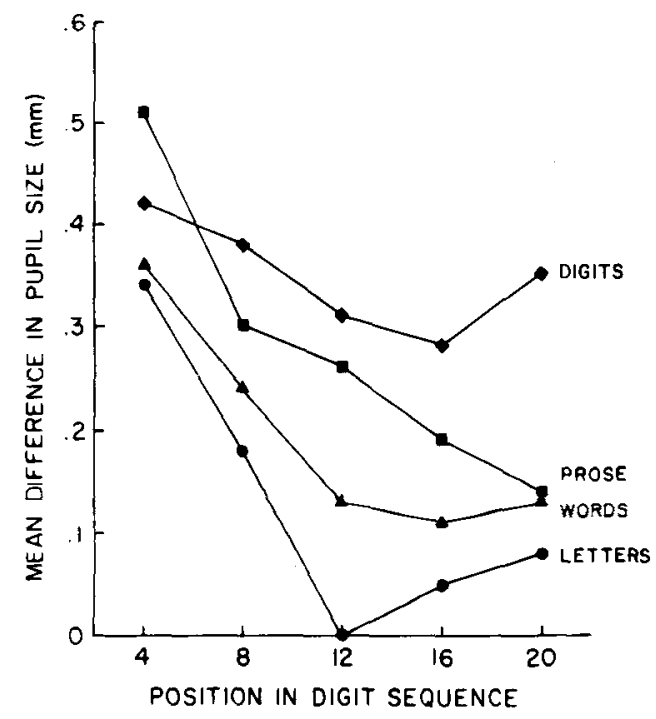

Figure 3. Mean difference from baseline in pupil size as a function of position of measurement in digit sequence, with digits as the primary message and digits, prose, words, or letters as the secondary message.
Table 4

Experiment IV: Percent Errors

\begin{tabular}{lcccr}
\hline & \multicolumn{4}{c}{ Quadrant } \\
\cline { 2 - 5 } $\begin{array}{l}\text { Unattended } \\
\text { Message }\end{array}$ & 1 & 2 & 3 & 4 \\
\hline Prose & 5.2 & 4.5 & 3.7 & 2.2 \\
Words & 3.7 & 5.9 & 6.7 & 3.7 \\
Digits & 5.9 & 9.6 & 7.4 & 14.1 \\
Letters & 5.9 & 2.9 & 2.9 & 10.4 \\
\hline
\end{tabular}

The within-subjects two-way analysis of variance (position by secondary message type) performed on the secondary verbal message data showed a significant effect of Type of Secondary Message $[F(3,24)=4.52$, $\mathrm{p}<.025, \mathrm{MSe}=.103]$ and of Position $[\mathrm{F}(4,32)=13.71$, $\mathrm{p}<.001, \mathrm{MSe}=.033$ ]. Most importantly, the interaction was also significant $[\mathrm{F}(12,96)=2.54, \mathrm{p}<.025$, $\mathrm{MSe}=.011]$. As in Experiment III, the interaction sum of squares was partitioned into two components, which showed that the digit curve interacted with the other three curves $[F(4,96)=4.51, p<.005, \mathrm{MSe}=.011]$, but the prose, word, and letter curves did not interact with each other $[F(8,96)=1.50, p>.05, \mathrm{MSe}=.011]$.

In order to locate the locus of the main effect for Tasks, a Scheffé post hoc analysis for within-subjects designs was performed. A comparison of the digit to the letter condition was not significant $[\mathrm{F}(1,24)=3.83$, $\mathrm{p}>.05, \mathrm{MSe}=.103]$. However, it was found that the prose and digit conditions combined were greater than the letter and word conditions combined $[F(1,24)=$ $4.77, \mathrm{p}<.05, \mathrm{MSe}=.103]$. No other differences close to significance were revealed.

An analysis of the shadowing errors across quadrants was performed as in Experiment III. The error data are shown in Table 4 . There was a significant main effect for Tasks $[F(3,24)=4.22, p<.025, \quad M S e=46.2]$. However, there was no effect for Quadrants $[F(3,24)<$ 1.0, $\mathrm{MSe}=65.6]$ and no interaction $[\mathrm{F}(9,72)=1.61$, $\mathrm{p}>.05, \mathrm{MSe}=43.8 \mathrm{]}$. A post hoc analysis showed that digits differed from prose, words, and letters combined $[F(1,24)=14.32, p<.01, \quad \mathrm{MSe}=46.2]$. No other differences were observed. In addition, an analysis of variance performed on the control condition, where subjects just listened to the secondary messages, showed no significant differences between the secondary messages $[F(3,24)<1.0, \mathrm{MSe}=.060]$.

\section{Discussion}

The results confirm the finding of Experiment III that the rate of decrease in shadowing difficulty across trials varies as a function of the categorical similarity of the primary and secondary messages. In Experiment IV where digits were shadowed, the slowest rate of decrease in pupil dilation over time was obtained for the digit secondary message.

The significant main effect for unattended messages was not observed in Experiment III. The contribution of the digits to this main effect is not unexpected be- 
cause task difficulty associated with digits was being maintained over time, while the difficulty of the other tasks was decreasing. No clear interpretation of the contribution of the prose message can be made. It is not due to the arousal content of the message, because no differences were found when subjects merely listened to secondary messages (control condition). However, an explanation in terms of cognitive complexity seems equally unlikely, because no differences were obtained in Experiment III and there was no difference between words and letters in Experiment IV. At this time, little importance can be attached to the secondary message main effect.

The error data also showed a main effect for secondary message, with digits resulting in more errors than the others. This is consistent with the finding that digits in the secondary message resulted in increased shadowing difficulty as measured by pupil dilation. However, the error data did not demonstrate the decrease in difficulty over time that pupil dilation indicated.

\section{GENERAL DISCUSSION}

Experiment I showed that pupil dilation measures the difficulty and effort involved in performing a shadowing task. In Experiment II it was shown that this difficulty was influenced by the content of the unattended message. Words in the secondary message resulted in more difficulty than did white noise. Furthermore, Experiment II showed that during a shadowing task pupil dilation decreased over time. This was interpreted to mean that the effort required and difficulty involved in shadowing decrease over time. In Experiments III and IV this rate of decrease was shown to be influenced by the categorical similarity of the attended and unattended messages. One way to explain this is by assuming that it takes time for the attentional mechanism to become focused on the primary message (Treisman et al., 1974), and maximal effort is involved in shadowing the beginning of the list. Furthermore, the ease with which the attentional mechanism can become focused on the attended message can be interfered with by categorical similarity between the attended and unattended messages. When the messages come from the same general semantic category, shadowing difficulty decreases little compared to when they come from different categories.

One possible explanation of the differential interference observed in these experiments for categorically similar vs. categorically dissimilar items is that there is a gradual reduction only in the semantic processing of the secondary message. This occurs when the secondary message is comprised of items which are categorically dissimilar to items in the primary message. However, this hypothesis can be rejected by noting that decreases in pupil size over time were observed even when white noise (i.e., nonsemantic material) was used as the unattended message.

One manner in which the data can be explained is to assume the processing system used in selective attention analyzes information in a hierarchical fashion. For example, the system would have information about whether an item was a letter or a digit before it had information about the particular letter or digit that was presented. The attentional mechanism can then use the results of this early categorical analysis as a basis for processing relevant and attenuating irrelevant messages. When both messages originate from the same category, this level of analysis is ineffective in distinguishing between relevant and irrelevant messages. This explanation is consistent with hierarchical properties of the attentional mechanism proposed by Treisman (1969) in her filter-attenuation theory of selective attention. However, the present data suggest that this hierarchical analysis be extended to the semantic level.

In order to account for the data using response selection theory (e.g., Deutsch \& Deutsch, 1963; Morton, 1969), it may be assumed that responses are arranged in a hierarchical manner. Using the logogen model (Morton, 1969) as an example, if the relevant message was comprised of digits, then response criteria for digit logogens could be lowered relative to the response criteria for nondigit logogens. This would effectively reduce the probability that competing responses to the irrelevant message would interfere with responses to the relevant message. However, when both relevant and irrelevant messages are composed of items from the same category, response criteria for relevant-item logogens cannot be lowered without concurrently lowering response criteria for irrelevant-item logogens. Under these conditions no improvement would be observed in the ability to isolate the response set associated with relevant items.

The present data can thus be interpreted within the framework of either filter-attenuation (Treisman, 1969) or response selection (Deutsch \& Deutsch, 1963; Morton, 1969). However, in either case, the present results suggest that characteristics of the attentional mechanism change over time.

The present results must be considered in relation to the finding of Treisman et al. (1974) that interference from synonyms in the secondary message decreased as shadowing continued. Certain evidence, which involves differential rates of decrease in shadowing difficulty, suggests that this synonym interference may be different from the interference resulting from categorical similarity. First, in the Treisman et al. (1974) study, while synonym interference decreased dramatically within $8 \mathrm{sec}$, vocal reaction time to words in the primary message increased over time. This was not due to the occurrence of a synonym embedded in the secondary list of words, since this effect was present 
both before and after a synonym occurred. Second, it was shown in Experiments III and IV that categorical interference remained quite stable and relatively high over a period of $20 \mathrm{sec}$ and, in particular, over the first $8 \mathrm{sec}$. Thus, the increase in vocal reaction time which Treisman et al. (1974) observed when both primary and secondary lists were composed of words parallels the present finding of sustained categorical interference when both primary and secondary messages were either letters or digits. It must be reiterated, though, that the Treisman et al. (1974) study was designed to investigate synonym interference. Consequently, no secondary messages were employed which contained items other than words. Thus, it is entirely possible that the observed increase in vocal reaction time as shadowing continued might also be observed when secondary messages comprised of items other than words are employed.

One further comment should be made about the error data reported in these experiments. The error data, which were relatively low, appeared to be insensitive to the differences in difficulty demonstrated by pupil dilation. This finding is consistent with the concept of "spare capacity" discussed by Kahneman (1973). When subjects perform shadowing tasks under conditions like those of the present experiments, they do not use all of the available capacity. When the shadowing task becomes more difficult due to interference from unattended messages, some of this spare capacity is demanded and used, thus preventing an increase in error rate but resulting in an increase in pupil dilation. It is quite possible that different results would be obtained if higher error rates were forced, since the attentional mechanism may operate differently at high vs. low levels of accuracy.

\section{REFERENCES}

Beatty, J., \& Kanneman, D. Pupillary changes in two memory tasks. Psychonomic Science, 1966, 5, 371-372.

Cortenn, R. S., \& DunN, D. Shock-associated words in a nonattended message: A test for momentary awareness. Journal of Experimental Psychology, 1974, 102, 1143-1144.
Corteen, R. S., \& Wood, B. Autonomic responses to shockassociated words in an unattended channel. Journal of Experimental Psychology, 1972, 94, 308-313.

Deutsch, J. A., \& Deutsch, D. Attention: Some theoretical considerations. Psychological Review, 1963, 70, 80-90.

Goldwater, B. C. Psychological significance of pupillary movements. Psychological Bulletin, 1972, 77, 340-355.

Hess, E. H., \& Polt, J. M. Pupil size in relation to mental activity during simple problem-solving. Science, 1964, 143, 1190-1192.

Kahneman, D. Attention and effort. Englewood Cliffs, N.J: Prentice-Hall, 1973.

Kahneman, D., \& Beatty, J. Pupil diameter and load on memory. Science, 1966, 154, 1583-1585.

LEwIS, J. Semantic processing of unattended messages using dichotic listening. Journal of Experimental Psychology, 1970, 85, 225-228.

Moray, N. Attention in dichotic listening: Affective cues and the influence of instructions. Quarterly Journal of Experimental Psychology, 1959, 11, 56-60.

Morton, J. Interaction of information in word recognition. Psychological Review, 1969, 76, 165-178.

Treisman, A. M. Strategies and models of selective attention. Psychological Review, 1969, 76, 282-299.

Treisman, A. M., Souire, R., \& Green, J. Semantic processes in dichotic listening? A replication. Memory \& Cognition, 1974, 2, 641-646.

ZeLniker, T. Perceptual attenuation of an irrelevant auditory verbal response in a selective attention task. Journal of Experimental Psychology, 1971, 87, 52-56.

\section{NOTES}

1. The data for one subject were not used in the analysis. Some of this subject's dilations were extremely large, being over 10 times greater than those of other subjects. We could not be sure that such dilations were not being caused by something other than an experimental variable. However, in all four experiments this was the only subject for whom this problem arose.

2. The noise condition was run merely to replicate the finding obtained in Experiment II that pupil dilation decreases over time in the presence of nonverbal unattended material. This position effect was obtained in both Experiment III and in Experiment IV $[F(4,36)=8.27, \quad \mathrm{p}<.001, \quad \mathrm{MSe}=.024$ and $\mathrm{F}(4,32)=5.05, \mathrm{p}<.005, \mathrm{MSe}=.017$, respectively $]$.

(Received for publication December 3, 1975; revision accepted March 19, 1976.) 